\title{
VOLKSWAGEN EN LA INDUSTRIA DE LOS AUTOMÓVILES. ALGUNAS APROXIMACIONES SOBRE LA TOMA DE DECISIONES DE NEGOCIO Y LA RESPONSABILIDAD SOCIAL EMPRESARIAL - CASO DIESELGATE.
}

\author{
Ricardo Adolfo Dizes Riveros \\ Facultad de Negocios, Gestión y Sostenibilidad \\ Especialización en Gestión Empresarial \\ rdizes@gmail.com
}

Diana Morales Ochoa

Especialización en Gestión Empresarial

Institución Universitaria Politécnico Grancolombiano

diana.morales.ochoa@hotmail.com

\author{
Allynson Venegas Camargo \\ Facultad de Negocios, Gestión y Sostenibilidad \\ Coordinadora Especialización en Gestión Empresarial \\ vcallynson@poligran.edu.co
}

Resumen

El presente artículo aborda desde una dimensión social, económica y medioambiental las implicaciones en las decisiones en el nivel directivo de empresas, tomando como referencia el caso "Diselgate" de Volkswagen, entorno a una estrategia empresarial basada en el crecimiento del mercado de automóviles desde el país de México hacia los Estados Unidos EEUU, donde su nivel de ingresos no representaba el 9\% de sus ventas totales, sobre la que utilizaron la adopción de motores Diesel eficientes y amigables con el medio ambiente en los vehículos de mostrador. Para promover esta imagen en el 2010, la compañía lanzó la campaña de marketing 
denominada "Think blue" que tenía por objetivo convertirla compañía en la ensambladora más ecológica y con mayor rendimiento por kilómetro recorrido.

El escrito hace referencia al estudio de caso, que en el año 2015 se desenmascaró uno de los mayores fraudes conocido en la historia automotriz este caso es conocido como "DIESELGATE”, la empresa Volkswagen instaló en sus vehículos un software que alteraba la lectura de emisión de gases para motores diésel, los cuales superaban el máximo permitido hasta en 40 veces. La universidad de West Virginia realizo muestreo sobre los vehículos y tomo las pruebas de campo que se presentaron ante los jueces para determinar el fraude el cual llego en Estados unidos a 17.500 millones de dólares como compensación a los propietarios de los 482.000 vehículos afectados, pagó al Departamento de Justicia una multa de 4.300 millones de dólares y una caída en la bolsa de inversión equivalente a 26.000 millones de euros.

El fraude, a pesar de descubrirse en los motores de vehículos en estados unidos, afectó a varios países y conllevo a replantear los controles a las empresas, con el compromiso del gobierno para garantizar que los ciudadanos como clientes no se vieran afectados o engañados con las compras legales que realizan en cada país.

La metodología usada es de orden cualitativo basado en el análisis de contenido documental especializado, seleccionado de diversas referencias y artículos del medio, de los gremios relacionados y publicaciones legales sobre las sentencias a los implicados

Las decisiones de los directores alineadas con la responsabilidad social empresarial generan valor y sostenibilidad de este a largo plazo, con serias consecuencias de orden corporativo e impacto en el consumidor final. Se recomienda que el caso sea punto de referencia en materia de buenas prácticas de manufactura para el sector y sea parte del ADN estratégico de las empresas, no solo en el ámbito de la planeación, sino, en la implementación misma para garantizar satisfacción de clientes y mayor generación de marca.

Palabras clave: Decisiones, responsabilidad social, impacto, medio ambiente, gerencia Introducción

El presente artículo pretende mostrar del caso "Dieselgate" para ilustrar como las decisiones de los directores influyeron en la aplicación de la Responsabilidad Social 
Empresarial y las consecuencias que estas traen en el entramado social, económico, laboral y medioambiental, que impacta no solo a la Organización, sino también a los grupos de interés y a la sociedad en general.

"Dieselgate" es el nombre popular del caso en el que la empresa Volkswagen (VW) en el año 2015 estuvo en el ojo del huracán por alterar los resultados de las pruebas de emisión de gases de sus modelos Jetta y Passsat, de motores Diésel en USA, que básicamente consistía en la manipulación del software de los vehículos aplicado a pruebas de laboratorio y de esta forma obtener la certificación sobre el cumplimiento de normas de emisión de gases, aval necesario para su comercialización en USA. En el contexto en el que se desarrolla el caso, se partirá de la historia y generalidades de la empresa, pasando por la descripción técnica del caso, para enmarcarlo en aproximaciones que tienen que ver con asuntos éticos, de responsabilidad Social y sus implicaciones.

Durante las últimas décadas, las prácticas de responsabilidad social de las empresas han venido en aumento, como parte del compromiso asumido ante generaciones futuras de construir una sociedad con mejores oportunidades, más equitativa, ambientalmente equilibrada y cada vez más comprometida con los derechos humanos. Las organizaciones cada vez se preocupan más por la sostenibilidad de los negocios, no solamente en satisfacer las necesidades de sus clientes, sino también en disminuir al máximo o eliminar el impacto negativo de su gestión tanto en el medio ambiente, como en la sociedad donde las organizaciones tienen influencia.

Antecedentes.

Historia de Volkswagen

El diseñador Ferdinand Porsche en 1930, creo un negocio con la idea de fabricar un coche pequeño y barato para vender en Alemania. Construyó el primer vehículo refrigerado por aire denominado Tipus 32, pero la planta que lo contrato quedo sin recursos y paro la producción.

Por referencias de Adolfo Hitler, se estableció la necesidad de construir un vehículo para la clase media alemana, capaz de cumplir con los estándares de la época y de fácil acceso, además de económico. Sobre este criterio surge la propuesta de la 
compañía Volkswagen, que en alemán traduce "Carro del pueblo" hacia otra compañía de la época, citando a Porsche a reunirse para diseñar el vehículo tipus 60, equipado con capacidad de transportar 4 personas, dotado de motor que funcionaba refrigerado por aire; esto, como respuesta a que en Estados Unidos Henry Ford y su modelo T tenia a la nación americana con el promedio más alto de vehículos por familia.

En septiembre de 1948, la responsabilidad era fabricar en México un solo modelo de automóvil sobre el que se permitieran en la industria llegar a una cifra por el orden de los 100 mil vehículos producidos y disponibles para la venta. En 1968 fue construida la fábrica en puebla, México y en 1972 superaron el record de fabricación de 15 millones convirtiéndose en el vehículo más fabricado de la historia. (DIAZ, 2015)

\section{Motores}

Técnicamente el motor Diesel fue inventado por el Ingeniero Rudolf Christian Karl Diesel y fue el primer motor de combustión interna en usar como combustible aceite mineral y fue presentado en la feria internacional de Paris en marzo de 1901. El año 1989 es un punto de referencia cuando Audi presenta en el Salón del Automóvil de Frankfurt un motor que combina la inyección directa y la sobrealimentación. Desde entonces es todo un referente en materia de Diésel. La tecnología TDI se ha extendido en estos años a todo tipo de vehículos dentro del grupo Volkswagen y Audi con precios más competitivos y unos niveles de emisiones muy bajos respecto a los modelos iniciales.

El principio de funcionamiento de un motor diésel es mediante la auto - ignición de la mezcla de aire-gas (combustible "pulverizado") se da por la alta temperatura a la que se hace llegar la mezcla por la elevación de la presión Dando como resultado, que el gas contenido en la cámara se expanda, impulsando el pistón hacia abajo. La biela transmite este movimiento al cigüeñal, para que este lo traslade a la trasmisión.

Para que se produzca la auto ignición se debe emplear gasóleo que es combustible más pesados que la gasolina, en 1 litro de gasóleo hay un 10\% más de energía calórica, estos combustibles nacen de la destilación del petróleo, por esta mayor energía calórica del combustible los motores diésel tiene un menor consumo de 
combustible, que los de gasolina, pero generan más partículas en suspensión en su ignición.

Tecnología TDI

En el año 1989 el Salón del Automóvil de Frankfurt Audi presenta un motor Diesel que combina la inyección directa y la sobrealimentación.

Audi presenta el primer motor que utilizaba la tecnología TDI Gracias a la utilización de la inyección directa y un turbocompresor alcanzaba una potencia de 120 $\mathrm{CV}$, con los que llegaba a los $200 \mathrm{~km} / \mathrm{h}$-frente a los $185 \mathrm{~km} / \mathrm{h}$ y se obtenía una reducción del consumo de combustible entre un 10 y un $15 \%$.

La tecnología TDI se ha extendido en estos años a todo tipo de vehículos dentro del grupo Volkswagen, Audi y Seat, con precios más competitivos y unos niveles de emisiones muy bajos respecto a los modelos creados inicialmente.

En los últimos 20 años se desarrollan motores con alto nivel de rendimiento y en la actualidad los motores TDI de Audi se componen de bloques de cuatro, seis, ocho y doce cilindros, con potencias entre 105 y $500 \mathrm{CV}$, Su aceptación es tan alta que son reconocidos por la suavidad de funcionamiento y el bajo nivel de ruido y vibraciones que se ha logrado. El desarrollo de otras marcas ha sido también determinante para evidenciar motores con mayor rendimiento y orientados a tener modelos y series de vehículos de más bajo costo, situación que no ocupa el tema central del presente caso.

Referente teórico.

Se produce el escándalo de emisiones contaminantes de vehículos de Volkswagen

En septiembre de 2015 se hizo pública la noticia de que la compañía de automóviles Volkswagen había instalado ilegalmente un software para alterar los resultados de los controles técnicos de emisiones contaminantes en 11 millones de automóviles con motor diésel, vendidos entre el año 2009 y el año 2015 alrededor del mundo. Como resultado de este fraude, sus motores superaban con éxito los estándares de la Agencia de Protección Ambiental de Estados Unidos 
(EPA). Los vehículos implicados emiten en condiciones reales hasta 40 veces el límite legal de óxidos de nitrógeno (Valentine, 2015).

La detección del problema comenzó a finales de 2012, cuando la organización sin ánimo de lucro International Council on Clean Transportation (ICCT) encargó a la West Virginia University para la realización de un estudio sobre las irregularidades en los reportes de las emisiones de vehículos diésel en las pruebas de campo, es decir, conduciendo el vehículo y simulando condiciones normales de manejo. Para ello, probaron emisiones de tres modelos diésel de Volkwagen tales como Passat, Jetta y BMW X5 (University, 2014).

Un equipo de profesores investigadores encabezado por Daniel Carder, dos profesores y un miembro de la facultad, efectuaron las pruebas de carretera en los alrededores de la ciudad de Los Ángeles, la Costa Oeste y Seattle. El estudio terminó en mayo de 2013 (Villaecija, 2015).

Otro auditor, Vicente Franco, se incorporó en 2013 al equipo de investigación del ICCT en Europa desde su sede en Berlín. Descubrieron que las emisiones de óxido de nitrógeno en los coches de Volkswagen probados multiplicaban entre 10 y 35 veces las registrados en el laboratorio durante la prueba de homologación. Los resultados se comunicaron en mayo de 2013 a la EPA, que realizó más estudios y destapó el escándalo en septiembre de 2015 (Jafee, 2015).

En octubre de 2016 la compañía Volkswagen pactó con las autoridades de Estados Unidos pagar 17.500 millones de dólares como compensación a los propietarios de los 482.000 vehículos afectados. En Estados Unidos pagó al Departamento de Justicia una multa de 4.300 millones de dólares y una caída en la bolsa de inversión equivalente a 26.000 millones de euros. (POZZI, VOLKSWAGEN TRUNCO SUS COCHES PARA EVITAR LOS LIMITES A LAS EMISIONES, 2015. P. 1).

Tabla 1. Mediciones de No. de los modelos Volkswagen Passat y Jetta

\begin{tabular}{|c|c|c|c|c|c|c|c|}
\hline \multirow{2}{*}{ Coche } & \multicolumn{3}{|c|}{ EPA (USA) } & \multicolumn{2}{|l|}{ Euro 5} & \multicolumn{2}{|l|}{$\begin{array}{l}\text { Euro } \\
6\end{array}$} \\
\hline & Limite & $\begin{array}{l}\text { Dinamó } \\
\text { metro }\end{array}$ & WVU & Limite & $\begin{array}{l}\text { Medida } \\
2011\end{array}$ & Limite & Medida \\
\hline
\end{tabular}




\begin{tabular}{llllllll}
\hline Vehiculo & & & & & & \\
A & 0,043 & 0,022 & $0,61-1,5$ & $\mathrm{O}, 18$ & $0,62+-$ & 0,016 & $\begin{array}{l}\text { Trampa } \\
\mathrm{d} \text { NOx } \\
\text { (Jetta) }\end{array}$ \\
& $\mathrm{g} / \mathrm{km}$ & $\mathrm{g} / \mathrm{km}$ & $\mathrm{g} / \mathrm{km}$ & $\mathrm{g} / \mathrm{km}$ & $0,19 \mathrm{~g} / \mathrm{km}$ & $\mathrm{g} / \mathrm{km}$ & (LNT) \\
& & & & & & & Reducci \\
& & & & & & ón \\
& & & & & catalític \\
Vehiculo & & & & & a \\
B & 0,043 & 0,016 & $0,34-0,067$ & $0,62+-$ & selectiv \\
& $\mathrm{g} / \mathrm{km}$ & $\mathrm{g} / \mathrm{km}$ & $\mathrm{g} / \mathrm{km}$ & $0,19 \mathrm{~g} / \mathrm{km}$ & a \\
(Passat) & & & & & basada \\
& & & & & & en urea \\
& & & & & (SCR) \\
\hline
\end{tabular}

Nota: Los modelos de los vehículos probados se reflejaban sin identificar en el estudio original.

Una investigación interna de Volkswagen concluyó que desde 2005 un grupo de ingenieros, al no lograr una solución técnica a tiempo y en presupuesto para superar los controles de emisiones de óxidos de nitrógeno establecidos en Estados Unidos, produjo un software que manipulaba los resultados al detectar las condiciones de realización de una prueba. Más tarde, la empresa logró solucionar el problema ingenieril, pero optó por mantener el uso del software. Por lo tanto, no se trató de un error sino de una cadena de actos erróneos que no fue interrumpida con el paso del tiempo (INC, 2018).

El 23 de septiembre de 2015, Martin Winterkorn, director ejecutivo de Volkswagen, Presento su renuncia tras 8 años de estar al frente de la empresa, debido al escándalo que ha afectado el prestigio de la compañía (REDACCION, 2015).

La responsabilidad de la dirección general quedo a cargo de Matthias Mueller anteriormente presidente y director ejecutivo de la marca Porsche (accionista mayoritario de VW). Los objetivos de Mueller al tomar posesión de su cargo eran recuperar la confianza del público, ofrecer una pronta solución al problema, además de reestructurar y reordenar la infraestructura para que no volviese a ocurrir un hecho de esta magnitud (CEST, 2018).

Desde septiembre de 2015 Volkswagen no vende coches diésel en Estados Unidos. 
En declaraciones de abril de 2017 Volkswagen dijo que venderá de nuevo modelos de la gama de 2015 en Estados Unidos que fueron recuperados, reparados y actualizados para cumplir con las normas anticontaminantes. No se espera que se lance algún modelo VW con motorización diésel antes de 2019, cuando VW decida si finalmente reiniciará la venta de dichos vehículos, ya que también inició un compromiso con la utilización de motores eléctricos.

Para compensar pérdidas, los modelos ya comercializados se les efectuaran las adecuaciones necesarias, mientras que los vehículos que queden en Stock que no haya sido posible vender y regresarlos a sus fábricas de origen. Se realizó un programa para exportarlos y venderlos en mercados donde el escándalo no haya afectado la imagen de la tecnología Diesel de la Marca (también igual se les efectuaran las reparaciones correspondientes) en algunos casos en mercados como México el precio de los mismos vehículos provenientes de Estados Unidos se redujo como parte de compensar las pérdidas y recobrar la imagen positiva que se tenía. igual los vehículos recuperados en Estados Unidos se volvieron a revender ya con las homologaciones correctas.

En 2015, en Estados Unidos los coches diésel suponían un 2\% del total de las ventas y dos de cada tres coches diésel eran Volkswagen. En Europa el 53\% de las ventas eran diésel y en España el 66\%.

Los modelos afectados son aquellos equipados con motores de la normativa Euro 5 entre el año 2009 y 2015. En la marca VW:

Polo, Golf, Touran, Passat, Scirocco, Toureg (2014 en EUU), Caddy, EOs, Transporter(t5), Jetta, Amarok, Sharn, Beetle,

Un estudio publicado en la revista Environmental Research Letters calculó en 59 el número de muertes prematuras causadas por el exceso de polución producido entre 2008 y 2015 en Estados Unidos. La mayor parte de estas muertes serían debidas a las partículas finas (87\%) y el resto debido al ozono (13\%). Según el estudio del International Institute for Applied Systems Analysis (IIASA) el fraude por las emisiones contaminantes provoca cada año 5000 muertes en Europa, lo que supone el aumento de la cifra de fallecimientos, de 5.270 a 9.830 en la Unión Europea, Noruega y Suiza.

(BARRET, 2015)

Ética y Responsabilidad Social 
El crecimiento en las empresas con la globalización es cada día mayor, por lo cual nacen nuevas necesidades como el compromiso de una organización ante los impactos que sus decisiones y actividades pueden ocasionar en el entorno donde influyen, mediante un comportamiento transparente deben contribuir al desarrollo sostenible de la sociedad que garantice una mejora, un crecimiento económico sin afectar la salud y el bienestar de la sociedad, tomado en cuenta las expectativas de los actores interesados, cumpliendo con la legislación a aplicar y las normas internacionales de comportamiento e integrada en la totalidad de la organización y se lleve a la practica en sus relaciones.

La ética es el mejor negocio porque orienta el comportamiento de las personas a realizar el bien, señala diversos caminos políticas y estrategias y ofrece los medios herramientas y prácticas para proceder rectamente en el negocio propio de cada empresa fomentando acciones sanas honestas y transparentes para lograr sus objetivos. Conviene insistir en que la ética no es tanto un discurso teórico o filosófico en torno a cómo deben ser las cosas desde un punto de vista ideal, sino un conjunto de actividades que reflejan un obrar correcto y que evitan conductas lesivas para personas y empresas (Yarse, 2016).

Una organización sólo puede sobrevivir a medio y largo plazo si resulta económicamente viable, es medioambientalmente sostenible y es socialmente responsable. Este es el planteamiento de la denominada 'Triple Bottom Line', introducida por John Elkington en su trabajo Cannibals with Forks (1997), en el sentido de atender las demandas de los diversos grupos de stakeholders, a través de la elaboración de la 'Triple Cuenta de Resultados', que comprende los resultados económicos, sociales y medioambientales de las compañías. (Orellana, 2006)

Para entender mejor la responsabilidad social, está el modelo tradicional de Triple cuenta de resultados autor de Jhon Elkinton 1994, que proponía que sus intereses económicos, sociales y ambientales podrían ser alienados en comportamientos éticos.

Tabla 2. El modelo tradicional de Triple cuenta de resultados.

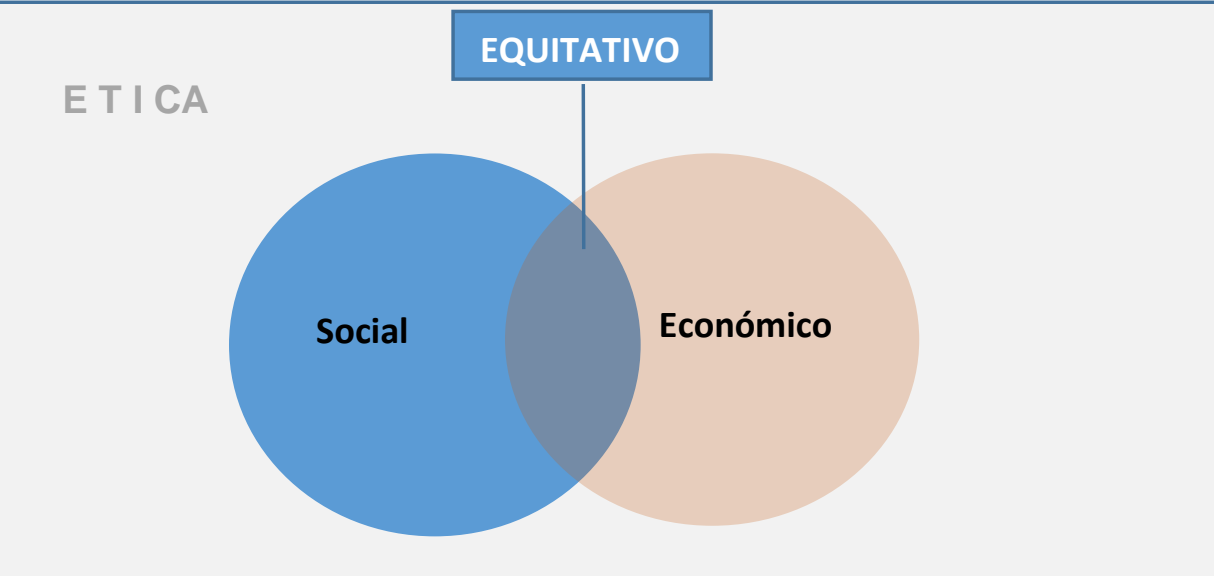




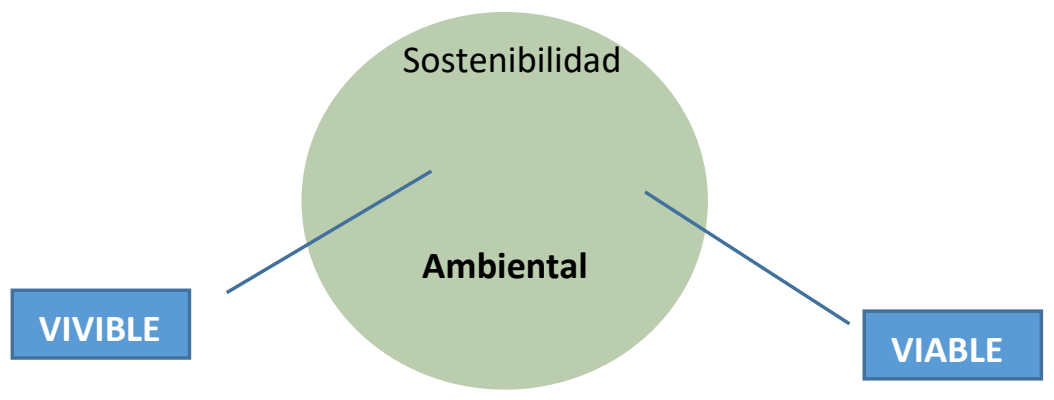

Fuente: los autores (2019)

Modelo de Jhon Elkinton, enmarcado en la ética.

La Responsabilidad de una organización se mide también ante los impactos que sus decisiones y actividades ocasionan en la sociedad y el medio ambiente, mediante un comportamiento ético y transparente que:

- Contribuye al desarrollo sostenible, incluyendo la salud y el bienestar de la sociedad

- Tome en consideración las expectativas de sus partes interesadas

Cumpla con la legislación aplicable y sea coherente con la normativa internacional de comportamiento

• Esté integrada en toda la organización y se lleve a la práctica en sus relaciones.” ISO26000

- Se establecen políticas las cuales deben manejar las empresas que buscan tener responsabilidad

En el campo de la responsabilidad social también podemos tomar en consideración los principios del Pacto global y derechos humanos de 1999 (Rulli \& Bautista, 2018).

Apreciamos como dentro del pacto mundial guía para las Empresas 4 de los 10 acuerdo fueron vulnerados por la decisión de los directores en el caso en mención.

\section{Derechos humanos:}

Primer Principio: Apoyar y respetar la protección de los derechos humanos fundamentales. 
Segundo Principio: Asegurar que sus empresas no son cómplices de la vulneración de los derechos humanos Laborales.

\section{Derechos Laborales:}

Tercer Principio: Apoyar la libertad de Asociación y el reconocimiento del derecho a la negociación colectiva.

Cuarto Principio: Promover la eliminación de toda forma de trabajo forzoso o realizado bajo coacción.

Quinto principio: Fomentar la erradicación del trabajo infantil.

Sexto Principio: Favorecer la abolición de las prácticas de discriminación en el empleo.

\section{Medio Ambiente:}

Séptimo Principio: Mantener un enfoque preventivo que favorezca el medio ambiente.

Octavo Principio: Promover las iniciativas que promuevan una mayor responsabilidad ambiental.

Noveno principio: Colaborar con el desarrollo y la difusión de las tecnologías respetuosas con el medio ambiente.

\section{Anticorrupción:}

Decimo Principio: Trabajar en contra de la corrupción en todas sus formas, incluidas la extorsión y el soborno.

Metodología

El enfoque de la investigación es de orden cualitativo, mediante un diseño de estudio de caso y basado en un contexto netamente descriptivo.

La investigación se llevó a cabo en tres momentos. Primero, desde un punto disciplinar se abordaron temas relacionados con el contexto, antecedentes y teoría relacionados con la empresa Volkswagen. En un segundo momento, se realizó una revisión de las implicaciones éticas y de responsabilidad social, con impacto social y económico. Finalmente, en un tercer momento se procedió al análisis, resultado de la observación realizada.

Discusión y Resultados 
Generar valor en una empresa con los principios de responsabilidad social es una dicotomía ya que los intereses particulares y de las naciones a veces van en contravía de la responsabilidad social, a mayores ventas más crecimiento de las fábricas lo que genera más empleo, impuestos y dinero circulante en la región donde está ubicada. El caso muestra como resultado que a pesar de las innumerables acciones para recuperar la imagen de la compañía tiempo después del escándalo, la función corporativa de la empresa nunca se recuperó, así como el precio de la acción de VW que no tuvo la capacidad de regresar a su máximo, aproximadamente en 250 euros, por el contrario, fue mucho menor ubicándose en promedio para los últimos 2 años en unos 150 euros, promedio aún inferior al presentado antes del escandala donde oscilaba en los 200 euros, tal y como se ilustra en el siguiente gráfico.

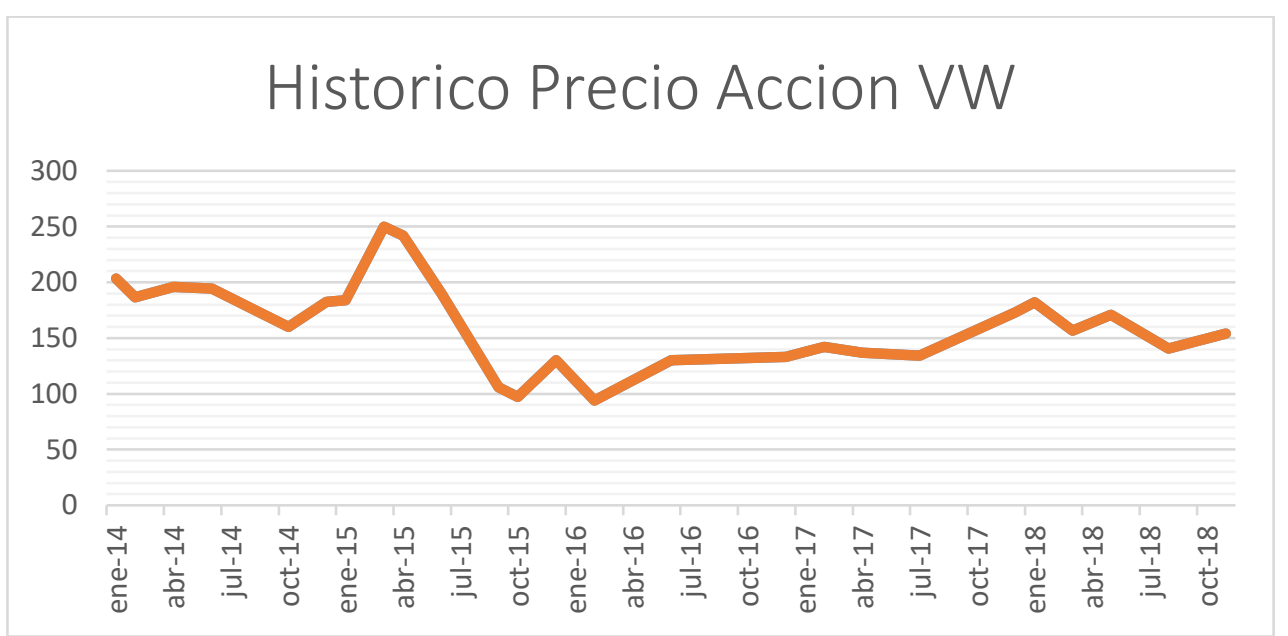

Gráfico 3. Histórico precio de la acción de Volkswagen

Fuente: los autores (2019)

Las decisiones de los directivos no estuvieron ligados a los valores de la empresa y por ende su estrategia no estuvo orientada a mejores prácticas, el distanciamiento de las decisiones de la promulgación de los valores corporativos implicara un impacto importante ya que no solo es cumplir los objetivos si no las acciones para que se cumplan.

Los atajos son los caminos más atractivos por ser fáciles, ya que implican menor esfuerzo, es decir, modificar un software es más rápido y económico que desarrollar un motor más eficiente y amigable con el medio ambiente, pero por los atajos se llega más rápido pero igualmente se corren mayores riesgos, tal como sucedió a la compañía. 
En el estudio del caso se evidencia como con las decisiones de los directores con implicaciones en principios y valores impactaron de manera importante el valor económico de la empresa, pero igualmente el entorno donde esta tiene influencia, poblaciones, grupos de interés y el medio ambiente. La responsabilidad social empresarial no es una práctica aparte de las mayores decisiones de la empresa, en sus diversas áreas funcionales tales como producción, comercialización, el componente humano y por supuesto en el nivel directivo; los resultados dependen de ello, la toma de decisiones de los directivos están alineadas con esta tendremos una sostenibilidad y relacionadas con nuestro entorno, por lo que se puede inferir que es una herramienta poderosa para cimentar la estrategia de la empresa.

En octubre de 2016 Volkswagen negoció con las autoridades de Estados Unidos pagar 17.500 millones de dólares como compensación a los propietarios de los 482.000 vehículos afectados, al Departamento de Justicia una multa de 4.300 millones de dólares y una caída en la bolsa de inversión equivalente a 26.000 millones de euros.

Para compensar pérdidas, los modelos ya comercializados se les efectuaran las adecuaciones necesarias, mientras que los vehículos que queden en Stock que no haya sido posible vender y regresarlos a sus fábricas de origen quedaran guardados en bodega hasta nueva instrucción. Un porcentaje de los vehículos recuperados en Estados Unidos se volvieron a revender ya con las homologaciones correctas y se le prohibió a VW exportar a otros países los vehículos que no cumplían con la norma (FUENTES, 2017).

Un estudio publicado en la revista Environmental Research Letters calculó en 59 el número de muertes prematuras causadas por el exceso de polución producido entre 2008 y 2015 por los vehículos trucados en los Estados Unidos. La mayor parte de estas muertes serían debidas a las partículas finas $(87 \%)$ y el resto debido al ozono (13\%). Si dichos vehículos hubieran cumplido la normativa desde finales de 2016 habrían evitado la muerte de otras 130 muertes prematuras. El número muy bajo de coches diésel en Estados Unidos, junto con la densidad de las ciudades europeas, quiere decir que la población europea está muchos más expuesta a la contaminación generada por el tráfico que la norteamericana. Según el estudio del International Institute for Applied Systems Analysis (IIASA) el fraude por las emisiones contaminantes provoca cada año 5000 muertes en Europa, lo que supone el aumento de la cifra de fallecimientos, de 5.270 a 9.830 en la Unión Europea, Noruega y Suiza. 
Con base en la discusión y resultados planteados se plantea la siguiente pregunta. ¿La presentación del caso sirve de referencia para otras empresas de la industria, inclusive para aquellas que pertenecen a otra industria?

A juicio de los autores, el caso hace referencia a una empresa e industria que suele ser sensible a cambios en los modelos de producción y comercialización, por lo que se hace necesario tener el presente como punto de referencia para efectos de estudio en los salones de clase y en las mesas de junta directiva. En este sentido, cabe resaltar que la discrecionalidad de la alta dirección se constituye fundamental para la toma de decisiones empresariales con enfoque a resultados.

\section{Conclusiones}

El caso es una ilustración de una problemática de negocio, interna, de un sector en el que las decisiones del nivel directivo traen consecuencias positivas o negativas, con un impacto en el medio que usa y comercializa vehículos. Es un modelo de prácticas inadecuadas en la realización de pruebas de calidad en la industria.

El impacto de las decisiones de los directores puede tener implicaciones jurídicas, ya que la corrupción según la definición de El profesor Petrus C. van Duyne; "La corrupción es una improbidad o deterioro en el proceso de toma de decisiones en el que un tomador de decisiones se desvía o exige desviación del criterio que debe regir su toma de decisiones, a cambio de una recompensa o por la promesa o expectativa de una recompensa. Si bien estos motivos influyen en su toma de decisiones no pueden ser parte (legítima) de la justificación de la decisión”. Es común ver la corrupción como el ejercicio de pago por algo, pero sirve de base para reflexionar ya que la recompensa puede ser el resultado que se busca para incrementar la remuneración de quienes ocupan el nivel directivo.

La Responsabilidad Social empresarial es una práctica que en teoría tiene como propósito realizar gestión y procesos de intervención en algunos grupos de interés, el caso de referencia es un ejemplo de lo que no se cumple sobre esta materia, debido a que, aunque en los pilares de la empresa Volkswagen están fundados en dicha práctica, sus directivos se desviaron de ello con las consecuencias ya vistas. 
La responsabilidad social Empresarial enmarcada en la "Tripe cuanta de resultados" de Jhon Elkinton de sostenibilidad y si las basamos en la Ética, daría como resultado lo que podemos definir como integralidad que deberá ser un derrotero o apoyo para la decisión de los directores.

La globalización y la diversidad de entidades a nivel mundial y local encargadas de controlar y regular las actividades de las multinacionales hace tentador por parte de los directores la toma de decisiones en contravía de la regulación.

Al momento de ser detectada la problemática de la compañía, las autoridades de Estados Unidos tanto regulatorias como judiciales implementaron las sanciones correspondientes con altas sumas económicas a la empresa y judiciales a algunos de los directivos involucrados y otros con investigaciones abiertas, lo anterior es un mensaje claro para que los directivos que se sientan tentados a este tipo de decisiones se abstengan de hacerlo. 


\section{REFERENCIAS}

Barret, S. R. (2015). Impact of The Volkswagen Emission Control Defeat Device On Us Public Health. Iop Science, p. 1.

Campos, A. (03 De 01 De 2011). Abc.Es. Obtenido De

Https://Www.Abc.Es/20110307/Motor-Reportajes/Abci-Volkswagen-Tecnologia-AnosMano-201103071137.Html.

Cest. (2018). Dimite El Presidente De Volkswagen. El País Economía, p. 1.

Diaz, C. T. (2015). Volkswagen La Historia. Milenio, p. 1.

Fuentes, V. (2017). Volkswagen Ya Puede Vender Esos Coches Diesel Que Almacena En Aparcamientos. Motor Pasión, p. 1.

Gonzalez, A. P. (2015). I3 Latam Y La Innovacion De Impacto. Unidos Innovando, 8. Inc, F. W. (21 De 10 De 2018). Wikipedia. Recuperado El 10 De 2018, De Wikipedia: Https://Es.Wikipedia.Org/Wiki/Esc\%C3\%A1ndalo_De_Emisiones_Contaminantes_De _Veh\%C3\%Adculos_Volkswagen

Jaffe, E. (2015). The Stady That Brought Down Volkswagen. Citylab, p. 1.

José Enrique Arias Pérez, C. A. (2011). Transferencia De Conocimiento Orientada A La Innovación Social En La Relación Ciencia, Tecnología Y Sociedad. Pensamiento \& Gestion, 137 - 166.

Pozzi, S. (19 De septiembre De 2015). Volkswagen Trunco Sus Coches Para Evitar Los Limites A Las Emisiones. El País, p. 1.

Pozzi, S. (10 De enero De 2017). Volkswagen Acuerda Una Multa De 4.100 Millones En Eeuu Por Ffraude De Las Emisiones. El País.

Redacción. (2015). Como Llego Volkswagen A Una De Las Peores Crisis De Su Historia. Bbc News, p. 1.

University, C. F. (2014). In Use Emission Testing of Ligth Duty Diesel Vehicles In The United States. Washington: Francisco Posada. 
Valentine, J. P. (2015, 09 18). Act Violations Carmaker Allegedly Used Software That Circumvent Emissions Testing for Software. Epa, p. 1.

Villaecija, R. (22 De 09 De 2015). Un Profesor De Universidad Y Una ONG

Destaparon El Escándalo De Volkswagen. El Mundo, p. 1. 